Supporting information

\title{
Polyacrylonitrile Nanofiber Membranes Modified with Ni-Based Conductive Metal Organic Frameworks for Air Filtration and Respiration Monitoring
}

Hansol Lee and Sangmin Jeon*

Department of Chemical Engineering, Pohang University of Science and Technology (POSTECH), Pohang, Gyeongbuk, Republic of Korea

*Author to whom correspondence should be addressed. E-mail: jeons@postech.ac.kr
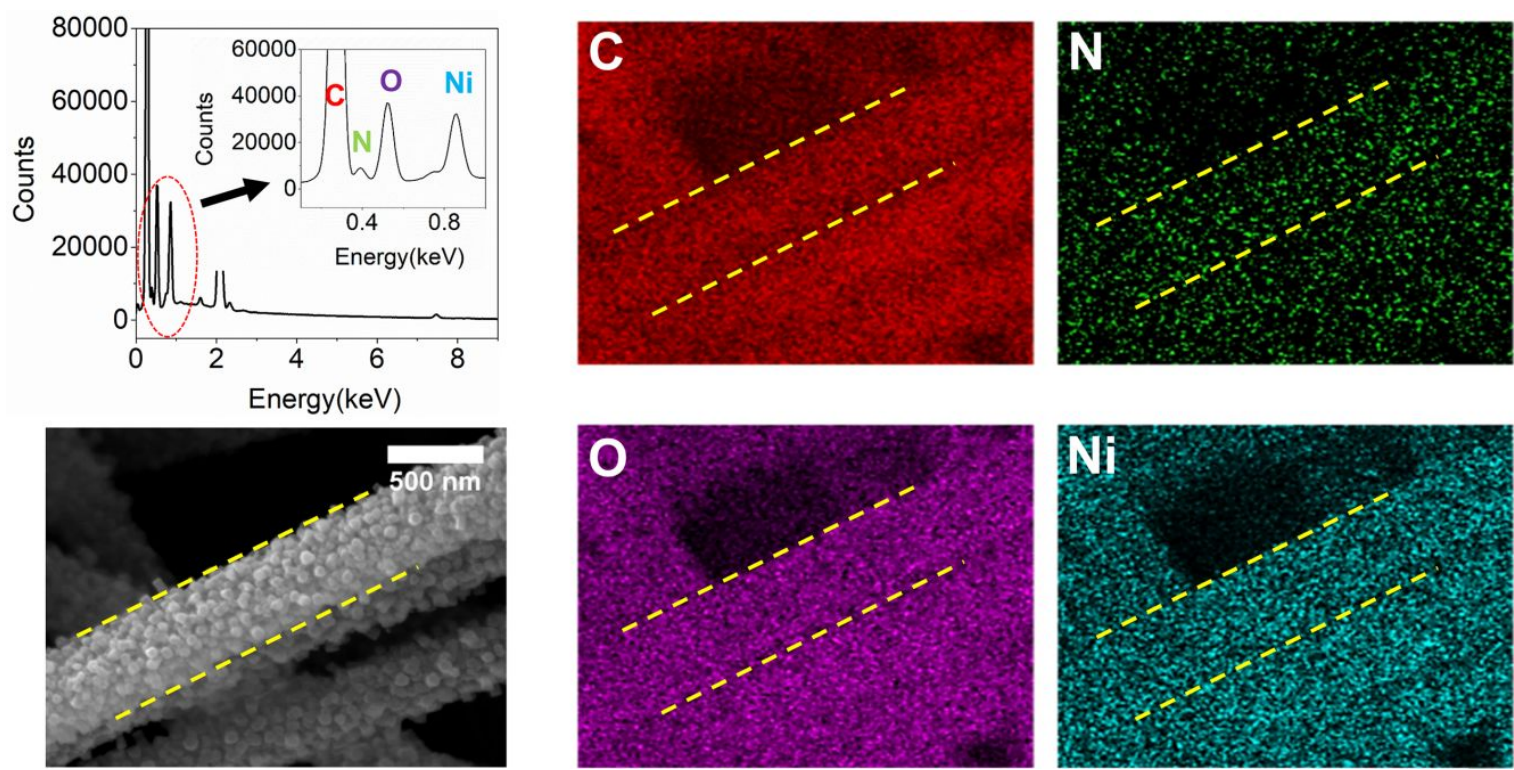

Figure S1. Energy dispersive X-ray spectra (EDS) elemental mappings of the nanofibers in PAN-MH3 after the growth of Ni-CAT-1 via a two-step hydrothermal reaction. 

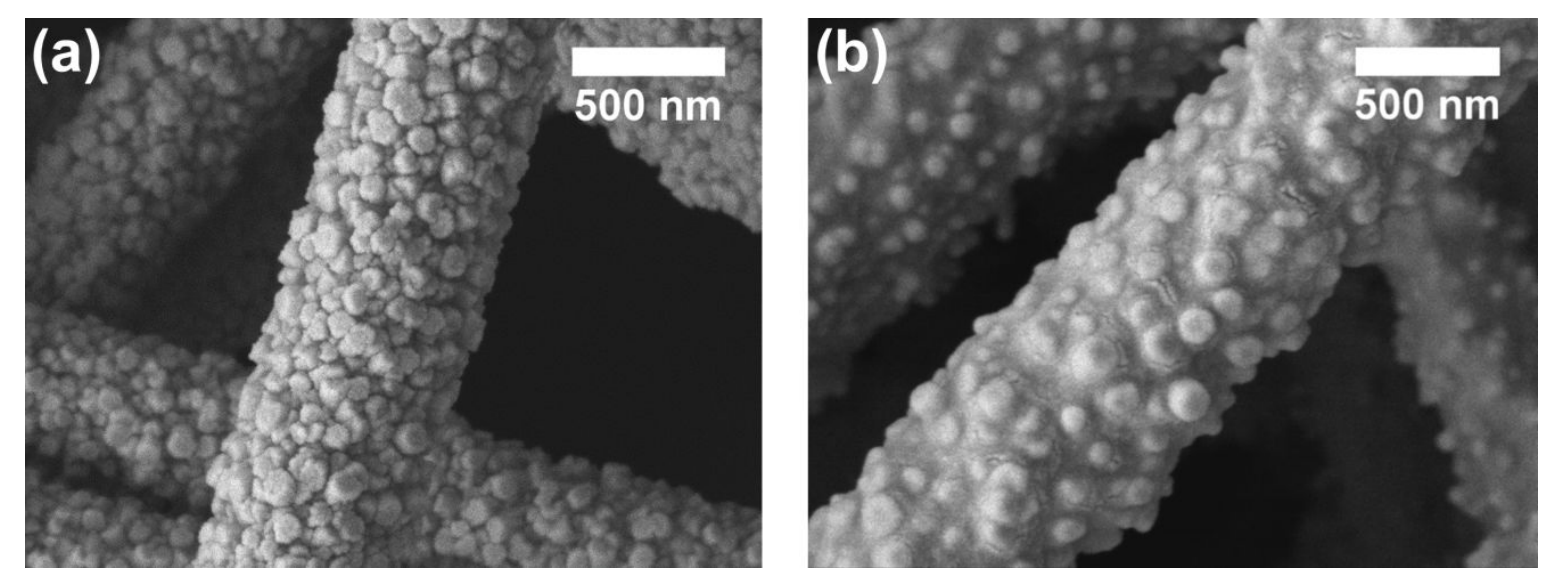

Figure S2. SEM images of PAN-MH3 (a) before and (b) after capturing the smoke from incense stick for $30 \mathrm{~s}$. 

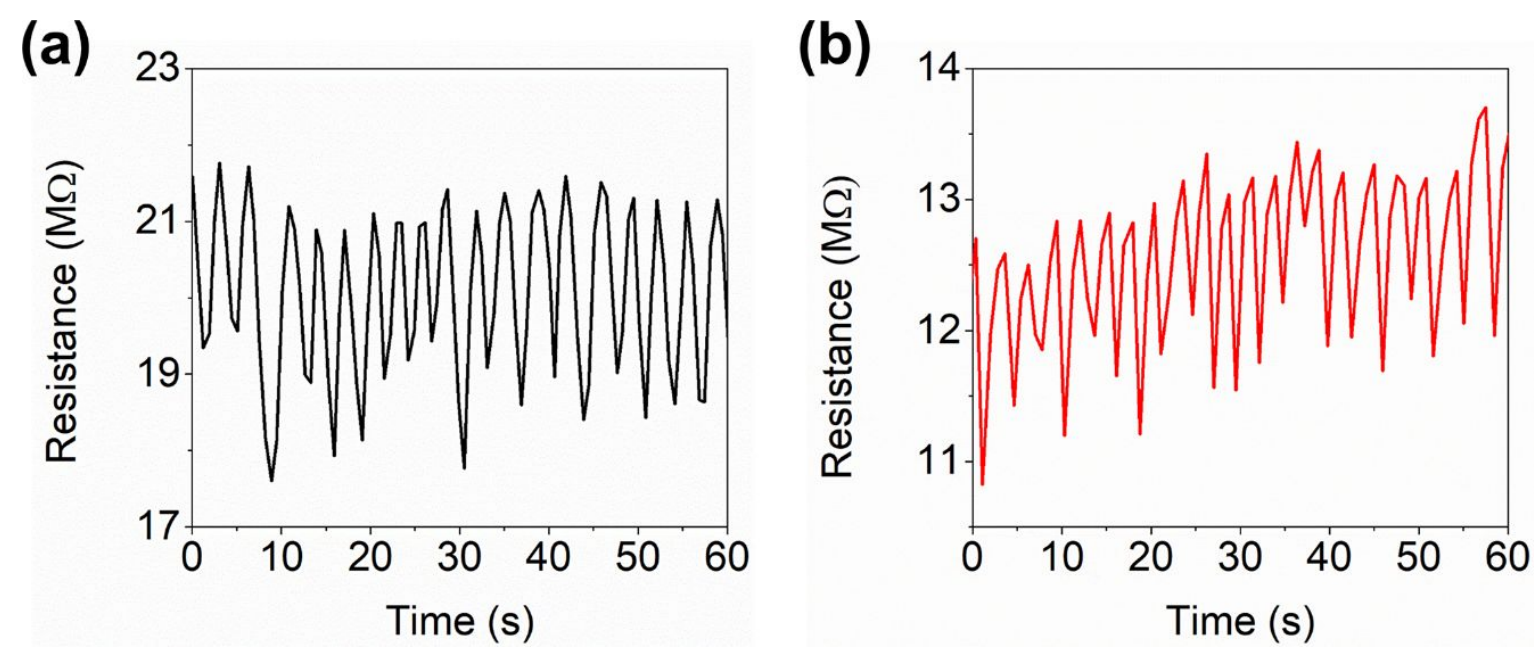

Figure S3. Real-time changes in electrical resistance of the PAN-MH3-attached face mask during respiration after loading (a) fine dusts and (b) smoke. 